\title{
DETERMINATION OF DISSOCIATION CONSTANTS OF MALONIC ACID IN (ETHYLENE GLYCOL-WATER)X\% MIXED SOLVENT AT DIFFERENT TEMPERATURES USING ELECTROMOTIVE FORCE MEASUREMENTS
}

\author{
Soleen Saeed Ahmed ${ }^{\mathrm{a}, *}$ and Lazgin A. Jamil ${ }^{\mathrm{b}}$ \\ ${ }^{\text {a }}$ Department of Chemistry, University of Zakho, Duhok, Kurdisatn Region, Iraq (soleen.saeed@uod.ac) \\ ${ }^{\mathrm{b}}$ Department of Chemistry, University of Zakho, Duhok, Kurdisatn Region, Iraq (Lazgin.jamil@uoz.edu.krd)
}

Received: Nov. 2018 / Accepted: Dec., 2018 / Published: Mar., 2019

https://doi.org/10.25271/siuoz.2019.7.1.573

\begin{abstract}
:
The first and second dissociation constants $\left(\mathrm{pK}_{1} \& \mathrm{pK}_{2}\right)$ of malonic acid in different composition of (ethylene glycol-water)\%, $(10,20$ and 30$) \%$ mixed solvent determined using the electromotive force measurements of galvanic cells without liquid junction at nine different temperatures, $(278.15-318.15) \mathrm{K}$ at $5^{\circ}$ interval, including the body temperature. The value of the first and second thermodynamic dissociation constants have been used to determine the thermodynamic quantities of two dissociation processes. These quantities involved the standard free energy, $\Delta \mathrm{G}^{0}$, standard enthalpy change, $\Delta \mathrm{H}^{0}$, standard entropy change, $\Delta \mathrm{S}^{0}$, and standard heat capacity change, $\Delta \mathrm{C}_{\mathrm{p}}{ }^{0}$.
\end{abstract}

KEYWORDS: Electromotive force, Thermodynamic dissociation constant, Malonic acid, Ethylene glycol-water mixed solvent, and thermodynamic quantity.

\section{INTRODUCTION}

Malonic acid (also called propanedioic acid) is one of saturated dicarboxylic acids and is homologous series of oxalic acid[1]. The general structure for these acids is $\mathrm{HOOC}-\left(\mathrm{CH}_{2}\right)_{\mathrm{n}}-$ $\mathrm{COOH}$, By using (x-ray) to obtain the crystal structure for malonic acid that has methylene group separating the carboxyl, the structure is $\mathrm{HOOC}\left(\mathrm{CH}_{2}\right)-\mathrm{COOH}$. Malonic acid itself is rather unstable and has few applications[2][3]. The reactivity of its methylene group provides the sequence of reactions of alkylation, hydrolysis of the esters and decarboxylation resulting in substituted ketones. The dissociation constant ratio $\mathrm{K}_{1} / \mathrm{K}_{2}$ is much higher than in disubstituted than in mono substituted malonic acid. Some evidence has accumulated that this is due to intermolecular hydrogen bonding in the disubstituted malonic acid mono anions. Intermolecular hydrogen bonding did not take place in the mono substituted malonic acid[4]. There are four General methods for the measurement of dissociation constant of carboxylic acid, including Potentiometric titration method[5][6][7][8], Conductometric method[9][10][11], Spectrophotometric methods[12][13][14], and Electromotive force measurement method[15][16][17]. There are many studies on dissociation constant of carboxylic acid in different organic-water mixed solvents and at different temperatures[18][19][20][21][22][23][24][25].

The present work involved the determination of the first and second dissociation constants $\left(\mathrm{pK}_{1} \& \mathrm{pK}_{2}\right)$ of malonic acid in different composition of ethylene glycol-water, (EG-water) mixed solvent, $(10 \%, 20 \%$ and $30 \%)$ using the electromotive force measurements of galvanic cells without liquid junction containing standard hydrogen electrode and silver-silver chloride electrode at different temperatures in the range $(278.15-318.15 \mathrm{~K})$ at $5^{\circ}$ interval. The value of the first and second thermodynamic dissociation constants have been used to determine the thermodynamic quantities of two dissociation processes. These quantities involved the standard free energy, $\Delta \mathrm{G}^{0}$, standard enthalpy change, $\Delta \mathrm{H}^{0}$, standard entropy change, $\Delta \mathrm{S}^{0}$, and standard heat capacity change, $\Delta \mathrm{C}_{\mathrm{p}}{ }^{0}$. However various studies employing electromotive measurement of cells without liquid junction using ethylene glycol-water mixture, there is still a need for investigation of this system with different dibasic carboxylic acid. In addition to its significance in understanding the role of high viscosity, density, as well as of low dielectric constant as compared with water on the phenomenon of solute-solute and solute-solvent interactions.

\section{EXPERIMENTAL}

All the chemicals used in this investigation were of analytical reagent grade purity. Malonic acid obtained from (SigmaAldrich, $\geq 99.95 \%$ ), was used without further purification. Ethylene glycol (analytical reagent, Merck, 99.5\%) was further purified by the method described earlier [26], freshly deionized water having a specific conductance of $0.2-0.9 \mu \mathrm{s} . \mathrm{cm}^{-1}$ was used.

\subsection{Cell A}

The first thermodynamic dissociation constant of malonic acid is studied by emf measurement of the galvanic cell without liquid junction. Such measurement is performed with a cell of type:

$$
\begin{array}{r}
\mathrm{Pt}_{(\mathrm{S})}, \mathbf{H}_{2(\mathrm{~g})}(\mathbf{1 a t m}) \mid \mathbf{H}_{2} \mathrm{Mal}_{\left(\mathrm{m}_{1}\right)}, \mathrm{NaHMal}_{\left(\mathrm{m}_{2}\right)}, \\
\mathrm{NaCl}_{\left(\mathrm{m}_{3}\right)} \text { in X\%EG }+\mathrm{H}_{2} \mathrm{O} \mid \operatorname{AgCl}_{(\mathrm{s})}, \mathbf{A g}_{(\mathrm{s})}
\end{array}
$$

Concentration of the malonic acid in the cell solution is $1 \mathrm{M}$, concentration of standard sodium hydroxide is $1 \mathrm{M}$ and concentration of sodium chloride is $0.5 \mathrm{M}$. Equal volumes of $1 \mathrm{M}$ sodium hydroxide with $1 \mathrm{M}$ of malonic acid were mixed to prepare a solution of $0.5 \mathrm{M}$ sodium salt of malonic acid. The different volume of $1 \mathrm{M}$ malonic acid, $0.5 \mathrm{M}$ sodium salt of malonic acid and $0.5 \mathrm{M}$ sodium chloride were mixed to prepare ten different molar concentrations of the malonic acid in cell solution in the range 0.01 to $0.1 \mathrm{M}$. These solutions prepared in $10 \%, 20 \%$ and $30 \%$ EG. $\%+\mathrm{H}_{2} \mathrm{O}$ mixed solvent.

\footnotetext{
* Corresponding author

This is an open access under a CC BY-NC-SA 4.0 license (https://creativecommons.org/licenses/by-nc-sa/4.0/)
} 


\subsection{Cell B}

$$
\begin{aligned}
& \mathbf{P t}_{(s)}, \mathbf{H}_{2(g)}(1 \mathrm{~atm})\left|\mathrm{NaHA}_{\left(\mathrm{m}_{1}\right)}, \mathrm{Na}_{2} \mathrm{~A}_{\left(\mathrm{m}_{2}\right)}, \mathrm{NaCl}_{\left(\mathrm{m}_{3}\right)}\right| \mathrm{AgCl}_{(\mathrm{s})} \\
& , \mathrm{Ag}_{(\mathrm{S})}
\end{aligned}
$$

In this cell, the concentration of the malonic acid is $1 \mathrm{M}$. Equal volumes of $1 \mathrm{M}$ malonic acid and $1 \mathrm{M}$ sodium hydroxide were mixed to prepare $0.5 \mathrm{M}$ HNaMal. And equal volumes of $1 \mathrm{M}$ malonic acid and $2 \mathrm{M}$ sodium hydroxide were mixed to prepare $0.5 \mathrm{M} \mathrm{Na}_{2} \mathrm{Mal}$. The other solution of this cell is sodium chloride with concentration $0.5 \mathrm{M}$, then the different volume of $0.5 \mathrm{M}$ HNaMal, $0.5 \mathrm{M} \mathrm{Na}_{2} \mathrm{Mal}$ and $0.5 \mathrm{M} \mathrm{NaCl}$ was mixed to prepare ten different molar concentrations of malonic acid. These solutions prepared in $10 \%, 20 \%$ and $30 \%$ EG. $\%$ - water mixed solvent.

\subsection{Electromotive force measurements}

The cell was, H-shape of all glass design, the dissolved air was removed from the cell by bubbling pure hydrogen which provided from electrolysis of acidified water [27] through the solution before the cell was filled. The cell was filled with solution and immersed in recirculating cooler for about 15 minute then prepared silver-silver chloride electrode [28][29] and standard hydrogen electrode[30][31] were dipped into the cell and pure hydrogen was passed (10-12 bubbles per second) through the electrode for about 1 hour to obtain stable emf the temperature was controlled to $\mp 0.1^{\circ} \mathrm{C}$ over the range $(278.15-318.15 \mathrm{~K})$. The barometric pressure was recorded within1.0 mmHg. The hydrogen gas was presaturated at the experimental temperature prior to being introduced into the cell. Then the e.m.f. of the cell was measured by digital multimeter (Auto Range AC DC LINI-T UT804), with an accuracy of $\mp 0.1 \mathrm{mV}$.

\section{RESULTS}

According to the Nernst equation

$\mathbf{E}=\mathbf{E}_{\mathbf{m}}^{\mathbf{0}}-\frac{\mathbf{R T}}{\mathbf{F}} \mathbf{L n \mathbf { a } _ { \pm }}$

The measurement of the electromotive force of each cell was corrected to a hydrogen partial pressure of latm in the usual way. The $\mathrm{pH}$ value of each buffer solutions at each temperature of measurement was estimated by the defining equation[32]

$$
-\log _{\mathrm{H}^{+}}=\frac{\left(E_{A}-\mathrm{E}_{\mathrm{m}}^{0}\right) F}{2.303 \mathrm{R} T}+\log \mathrm{Cl}_{\mathrm{Cl}^{-}}-2 \log \gamma_{\mathrm{H}^{+}}
$$

Where $\gamma$ represents a molal activity coefficient; $E_{A}$ and $\mathrm{E}_{\mathrm{m}}^{0}$ are the electromotive force and standard electromotive force, respectively of the cell $\mathrm{A}$ at the appropriate absolute temperature, $\mathrm{R}$ and $\mathrm{F}$ are the gas constant and the faraday respectively. Values of $\mathrm{E}_{\mathrm{m}}^{0}$ and $2.3026 \mathrm{RT} / \mathrm{F}$ in absolute volts are summarized in an earlier publication[33].

The ionic strength $\left(\mathrm{I}_{1}\right)$ of the solution in the cell A [25] is given by equation (3)

$$
\mathbf{I}_{1}=\mathbf{m}_{\mathrm{H}^{+}}+\mathbf{m}_{2}+\mathbf{m}_{3}
$$

From dissociation of $\mathrm{H}_{2} \mathrm{Mal}$ in different solvents composition, 10,20 , and $30 \%$ ethylene glycol-water mixed solvent, the apparent first dissociation constant $\mathrm{p} \overline{\mathrm{K}}_{1}$, was calculated from equation[23]

$$
\frac{\mathbf{E}_{\mathbf{A}}-\mathbf{E}_{\mathbf{m}}^{\mathbf{0}}}{\mathbf{K}}+\log \frac{\mathbf{m}_{\mathbf{3}}\left(\mathbf{m}_{\mathbf{1}}-\mathbf{m}_{\mathbf{H}^{+}}\right)}{\left(\mathbf{m}_{\mathbf{2}}+\mathbf{m}_{\mathbf{H}^{+}}\right)}=-\boldsymbol{\beta} \mathbf{I}-\log \mathrm{K}_{\mathbf{1}}
$$

Where the left hand side of eq (4) is the apparent first dissociation constant $\overline{\mathrm{K}}_{1},\left(-\log \mathrm{K}_{1}\right)$ of malonic acid and $\mathrm{pK}_{1}$ is the intercept of the plot between the $\mathrm{p} \overline{\mathrm{K}}_{1}$ (y axis) with $\mathrm{I}_{1}$ (x axis).

Table (1) shows the values of $\mathrm{m}_{\mathrm{H}^{+}}$of solution of the cell A. The table (2) shows ionic strength $\mathrm{I}_{1}$ of solution of the cell A. The thermodynamic first dissociation constants, is obtained by a linear methods using (L.S.M) method of $p \bar{K}_{1}$ to $I_{1}=0$, according to the equation (4), and are listed in table (3). Thermodynamic functions of first dissociation constants $\mathrm{pK}_{1}$ at different temperatures with other constants in $\mathrm{X} \%$ Ethylene glycol-water mixtures are listed in tables (4). The values of $\mathrm{pK}_{1}$ increase (acidity decreases) as mole fraction of Ethylene glycol increases in mixture with water because the solvation of the proton and intra-hydrogen bonding with the mono anion would be both hindered appreciably by the addition of larger and more viscous Ethylene glycol molecules. Hence a decrease acidity of

\begin{tabular}{|c|c|c|c|c|c|c|c|c|c|}
\hline \multicolumn{10}{|c|}{$10 \%$ EG } \\
\hline \multirow{2}{*}{$\begin{array}{c}\text { Concentration } \\
\mathrm{m}\end{array}$} & \multicolumn{9}{|c|}{ Temperature ${ }^{\circ} \mathrm{C}$} \\
\hline & 278.15 & 283.15 & 288.15 & 293.15 & 298.15 & 303.15 & 308.15 & 313.15 & 318.15 \\
\hline 0.009979 & 0.1590 & 0.1636 & 0.1668 & 0.1719 & 0.1820 & 0.1843 & 0.1859 & 0.1989 & 0.2010 \\
\hline 0.019979 & 0.2194 & 0.2426 & 0.2620 & 0.2867 & 0.2961 & 0.3010 & 0.3127 & 0.3233 & 0.3314 \\
\hline 0.030000 & 0.3144 & 0.3128 & 0.3359 & 0.2793 & 0.3254 & 0.3401 & 0.3604 & 0.3700 & 0.3852 \\
\hline 0.040042 & 0.2740 & 0.2861 & 0.3246 & 0.3401 & 0.2468 & 0.2779 & 0.3024 & 0.3222 & 0.3352 \\
\hline 0.050104 & 0.2295 & 0.2383 & 0.2648 & 0.2907 & 0.2888 & 0.3050 & 0.3097 & 0.3298 & 0.3505 \\
\hline 0.060188 & 0.2471 & 0.2837 & 0.2920 & 0.3249 & 0.3373 & 0.3509 & 0.3745 & 0.3828 & 0.4363 \\
\hline 0.070292 & 0.2932 & 0.3050 & 0.3169 & 0.3314 & 0.3461 & 0.3764 & 0.4129 & 0.4467 & 0.5035 \\
\hline 0.080418 & 0.2664 & 0.2783 & 0.3010 & 0.3170 & 0.3256 & 0.3778 & 0.4756 & 0.4902 & 0.5631 \\
\hline 0.090565 & 0.3022 & 0.3131 & 0.3213 & 0.3456 & 0.3565 & 0.4170 & 0.4981 & 0.5597 & 0.6267 \\
\hline 0.100733 & 0.3303 & 0.3494 & 0.3629 & 0.3720 & 0.3962 & 0.4494 & 0.4926 & 0.6267 & 0.7144 \\
\hline $\mathbf{m}$ & \multicolumn{9}{|c|}{$20 \%$ EG } \\
\hline 0.009726 & 0.0988 & 0.1125 & 0.1192 & 0.1322 & 0.1351 & 0.1411 & 0.1451 & 0.1468 & 0.1507 \\
\hline 0.019472 & 0.1597 & 0.1557 & 0.1659 & 0.1561 & 0.1727 & 0.1770 & 0.1854 & 0.1961 & 0.2040 \\
\hline 0.029238 & 0.1873 & 0.2048 & 0.2224 & 0.2333 & 0.2560 & 0.2595 & 0.2638 & 0.2622 & 0.2733 \\
\hline 0.039023 & 0.2027 & 0.2093 & 0.2320 & 0.2542 & 0.2778 & 0.2879 & 0.2992 & 0.3129 & 0.3139 \\
\hline 0.048829 & 0.1941 & 0.2123 & 0.2449 & 0.2526 & 0.2676 & 0.2828 & 0.3076 & 0.3179 & 0.3318 \\
\hline 0.058654 & 0.1813 & 0.2110 & 0.2563 & 0.2588 & 0.3017 & 0.3407 & 0.3488 & 0.3690 & 0.3982 \\
\hline 0.068499 & 0.1979 & 0.2231 & 0.2577 & 0.2835 & 0.3133 & 0.3599 & 0.3935 & 0.4013 & 0.4241 \\
\hline 0.078365 & 0.1923 & 0.2398 & 0.2517 & 0.2755 & 0.3457 & 0.3640 & 0.3927 & 0.4290 & 0.4607 \\
\hline 0.088250 & 0.2200 & 0.2466 & 0.2787 & 0.2909 & 0.3502 & 0.3911 & 0.4256 & 0.4792 & 0.5089 \\
\hline 0.098156 & 0.2335 & 0.2630 & 0.2857 & 0.3132 & 0.3463 & 0.4057 & 0.4623 & 0.5056 & 0.6039 \\
\hline $\mathbf{m}$ & \multicolumn{9}{|c|}{$30 \%$ EG } \\
\hline 0.009608 & 0.0898 & 0.0932 & 0.0990 & 0.1021 & 0.1036 & 0.1062 & 0.1056 & 0.1074 & 0.1104 \\
\hline
\end{tabular}
malonic acid is expected to continue as mole fraction of Ethylene glycol increases.

Table 1. Concentration of hydrogen ion $\mathrm{m}_{\mathrm{H}}$ for different concentrations of malonic acid in 10, 20, and 30\% Ethylene glycolwater mixtures at different temperatures. $\mathrm{m}_{\mathrm{H}}=10^{-2}$ 


\begin{tabular}{|l|l|l|l|l|l|l|l|l|l|}
\hline 0.019236 & 0.1188 & 0.1217 & 0.1257 & 0.1317 & 0.1340 & 0.1390 & 0.1439 & 0.1445 & 0.1441 \\
\hline 0.028882 & 0.1194 & 0.1279 & 0.1355 & 0.1411 & 0.1484 & 0.1541 & 0.1603 & 0.1636 & 0.1699 \\
\hline 0.038548 & 0.1320 & 0.1395 & 0.1508 & 0.1556 & 0.1616 & 0.1677 & 0.1719 & 0.1766 & 0.1888 \\
\hline 0.048234 & 0.1222 & 0.1309 & 0.1388 & 0.1468 & 0.1563 & 0.1654 & 0.1760 & 0.1883 & 0.2093 \\
\hline 0.057939 & 0.1251 & 0.1378 & 0.1488 & 0.1565 & 0.1650 & 0.1749 & 0.1879 & 0.1970 & 0.2155 \\
\hline 0.067663 & 0.1123 & 0.1212 & 0.1310 & 0.1362 & 0.1466 & 0.1641 & 0.1723 & 0.1982 & 0.2287 \\
\hline 0.077407 & 0.1161 & 0.1271 & 0.1381 & 0.1456 & 0.1556 & 0.1805 & 0.1897 & 0.2065 & 0.2360 \\
\hline 0.087171 & 0.1237 & 0.1389 & 0.1451 & 0.1562 & 0.1778 & 0.2008 & 0.2110 & 0.2248 & 0.2704 \\
\hline 0.096954 & 0.1421 & 0.1500 & 0.1606 & 0.1757 & 0.1915 & 0.2115 & 0.2328 & 0.2434 & 0.2794 \\
\hline
\end{tabular}

Table 2. Ionic strength $\mathbf{I}_{\mathbf{1}}$ at different molal concentrations from the cell A in 10, 20, and 30\% Ethylene glycol-water mixtures at different temperatures. $\mathbf{I}_{1}=\mathbf{1 0}^{-3}$

\begin{tabular}{|c|c|c|c|c|c|c|c|c|c|}
\hline \multicolumn{10}{|c|}{$10 \%$ EG } \\
\hline Concentration & \multicolumn{9}{|c|}{ Temperature ${ }^{\circ} \mathrm{C}$} \\
\hline $\mathbf{m}$ & 278.15 & 283.15 & 288.15 & 293.15 & 298.15 & 303.15 & 308.15 & 313.15 & 318.15 \\
\hline 0.009979 & 21.546 & 21.592 & 21.624 & 21.675 & 21.776 & 21.799 & 21.815 & 21.945 & 21.966 \\
\hline 0.019979 & 42.143 & 42.375 & 42.569 & 42.815 & 42.910 & 42.958 & 43.076 & 43.182 & 43.263 \\
\hline 0.030000 & 63.123 & 63.106 & 63.338 & 62.772 & 63.233 & 63.380 & 63.582 & 63.679 & 63.830 \\
\hline 0.040042 & 82.785 & 82.906 & 83.291 & 83.446 & 82.513 & 82.824 & 83.069 & 83.267 & 83.397 \\
\hline 0.050104 & 102.44 & 102.53 & 102.797 & 103.06 & 103.04 & 103.20 & 103.25 & 103.45 & 103.65 \\
\hline 0.060188 & 122.76 & 123.13 & 123.210 & 123.54 & 123.66 & 123.80 & 124.03 & 124.12 & 124.65 \\
\hline 0.070292 & 143.40 & 143.52 & 143.638 & 143.78 & 143.93 & 144.23 & 144.60 & 144.94 & 145.50 \\
\hline 0.080418 & 163.35 & 163.47 & 163.694 & 163.86 & 163.94 & 164.46 & 165.44 & 165.59 & 166.32 \\
\hline 0.090565 & 183.96 & 184.07 & 184.152 & 184.39 & 184.50 & 185.11 & 185.92 & 186.54 & 187.21 \\
\hline 0.100733 & 204.53 & 204.72 & 204.858 & 204.95 & 205.19 & 205.72 & 206.16 & 207.50 & 208.37 \\
\hline $\mathbf{m}$ & \multicolumn{9}{|c|}{$20 \%$ EG } \\
\hline 0.009726 & 20.438 & 20.576 & 20.642 & 20.772 & 20.801 & 20.862 & 20.901 & 20.918 & 20.957 \\
\hline 0.019472 & 40.532 & 40.492 & 40.595 & 40.497 & 40.662 & 40.705 & 40.789 & 40.896 & 40.975 \\
\hline 0.029238 & 60.328 & 60.504 & 60.679 & 60.788 & 61.016 & 61.050 & 61.094 & 61.078 & 61.189 \\
\hline 0.039023 & 80.038 & 80.103 & 80.330 & 80.553 & 80.789 & 80.890 & 81.003 & 81.140 & 81.149 \\
\hline 0.048829 & 99.542 & 99.724 & 100.05 & 100.13 & 100.28 & 100.43 & 100.68 & 100.78 & 100.92 \\
\hline 0.058654 & 119.04 & 119.34 & 119.79 & 119.82 & 120.24 & 120.64 & 120.72 & 120.92 & 121.21 \\
\hline 0.068499 & 138.87 & 139.12 & 139.47 & 139.72 & 140.02 & 140.49 & 140.82 & 140.90 & 141.13 \\
\hline 0.078365 & 158.51 & 158.98 & 159.10 & 159.34 & 160.04 & 160.23 & 160.51 & 160.88 & 161.19 \\
\hline 0.088250 & 178.52 & 178.78 & 179.11 & 179.23 & 179.82 & 180.23 & 180.57 & 181.11 & 181.41 \\
\hline 0.098156 & 198.42 & 198.72 & 198.94 & 199.22 & 199.55 & 200.14 & 200.71 & 201.14 & 202.13 \\
\hline $\mathbf{m}$ & \multicolumn{9}{|c|}{$30 \%$ EG } \\
\hline 0.009608 & 20.112 & 20.146 & 20.205 & 20.236 & 20.250 & 20.277 & 20.271 & 20.288 & 20.318 \\
\hline 0.019236 & 39.650 & 39.680 & 39.719 & 39.779 & 39.802 & 39.852 & 39.902 & 39.908 & 39.903 \\
\hline 0.028882 & 58.939 & 59.024 & 59.100 & 59.156 & 59.229 & 59.285 & 59.348 & 59.381 & 59.444 \\
\hline 0.038548 & 78.381 & 78.457 & 78.569 & 78.617 & 78.678 & 78.739 & 78.780 & 78.828 & 78.950 \\
\hline 0.048234 & 97.635 & 97.722 & 97.801 & 97.881 & 97.976 & 98.067 & 98.173 & 98.296 & 98.506 \\
\hline 0.057939 & 117.05 & 117.18 & 117.29 & 117.36 & 117.45 & 117.55 & 117.68 & 117.77 & 117.95 \\
\hline 0.067663 & 136.34 & 136.43 & 136.53 & 136.58 & 136.69 & 136.86 & 136.94 & 137.20 & 137.51 \\
\hline 0.077407 & 155.84 & 155.95 & 156.06 & 156.13 & 156.23 & 156.48 & 156.57 & 156.74 & 157.03 \\
\hline 0.087171 & 175.40 & 175.55 & 175.62 & 175.73 & 175.94 & 176.17 & 176.27 & 176.41 & 176.87 \\
\hline 0.096954 & 195.11 & 195.19 & 195.30 & 195.45 & 195.60 & 195.80 & 196.02 & 196.12 & 196.48 \\
\hline
\end{tabular}

Table 3. Apparent first dissociation constant $\mathbf{p} \overline{\mathbf{K}}_{\mathbf{1}}$ at different temperatures and molal concentrations in 10, 20, and $30 \%$ Ethylene glycol-water mixtures.

\begin{tabular}{|c|c|c|c|c|c|c|c|c|c|}
\hline \multicolumn{10}{|c|}{$10 \%$ EG } \\
\hline \multirow{2}{*}{$\frac{\text { Concentration }}{\mathrm{m}}$} & \multicolumn{9}{|c|}{ Temperature ${ }^{\circ} \mathrm{C}$} \\
\hline & 278.15 & 283.15 & 288.15 & 293.15 & 298.15 & 303.15 & 308.15 & 313.15 & 318.15 \\
\hline 0.009979 & 2.6953 & 2.6592 & 2.6342 & 2.6151 & 2.6073 & 2.6096 & 2.6027 & 2.5873 & 2.5865 \\
\hline 0.019979 & 2.8193 & 2.7863 & 2.7418 & 2.7339 & 2.7344 & 2.7418 & 2.7327 & 2.7338 & 2.7296 \\
\hline 0.030000 & 2.8622 & 2.8495 & 2.8125 & 2.8342 & 2.8342 & 2.8329 & 2.8266 & 2.8380 & 2.8298 \\
\hline 0.040042 & 3.0047 & 2.9909 & 2.9350 & 2.9510 & 2.9800 & 2.9733 & 2.9729 & 2.9798 & 2.9752 \\
\hline 0.050104 & 3.1069 & 3.0892 & 3.0444 & 3.0509 & 3.0603 & 3.0628 & 3.0754 & 3.0752 & 3.0716 \\
\hline 0.060188 & 3.1795 & 3.1404 & 3.1048 & 3.1114 & 3.1190 & 3.1278 & 3.1315 & 3.1387 & 3.1265 \\
\hline 0.070292 & 3.2135 & 3.1930 & 3.1556 & 3.1690 & 3.1768 & 3.1874 & 3.1869 & 3.1866 & 3.1817 \\
\hline 0.080418 & 3.2646 & 3.2445 & 3.2029 & 3.2150 & 3.2291 & 3.2380 & 3.2267 & 3.2389 & 3.2175 \\
\hline 0.090565 & 3.2958 & 3.2727 & 3.2437 & 3.2450 & 3.2611 & 3.2718 & 3.2620 & 3.2702 & 3.2585 \\
\hline 0.100733 & 3.3349 & 3.3043 & 3.2664 & 3.2783 & 3.2918 & 3.3040 & 3.2952 & 3.2916 & 3.2823 \\
\hline $\mathbf{m}$ & \multicolumn{9}{|c|}{$20 \%$ EG } \\
\hline 0.009726 & 2.6953 & 2.6592 & 2.6342 & 2.6151 & 2.6073 & 2.6096 & 2.6027 & 2.5873 & 2.5865 \\
\hline 0.019472 & 2.8193 & 2.7863 & 2.7418 & 2.7339 & 2.7344 & 2.7418 & 2.7327 & 2.7338 & 2.7296 \\
\hline
\end{tabular}




\begin{tabular}{|c|c|c|c|c|c|c|c|c|c|}
\hline 0.029238 & 2.8622 & 2.8495 & 2.8125 & 2.8342 & 2.8342 & 2.8329 & 2.8266 & 2.8380 & 2.8298 \\
\hline 0.039023 & 3.0047 & 2.9909 & 2.9350 & 2.9510 & 2.9800 & 2.9733 & 2.9729 & 2.9798 & 2.9752 \\
\hline 0.048829 & 3.1069 & 3.0892 & 3.0444 & 3.0509 & 3.0603 & 3.0628 & 3.0754 & 3.0752 & 3.0716 \\
\hline 0.058654 & 3.1795 & 3.1404 & 3.1048 & 3.1114 & 3.1190 & 3.1278 & 3.1315 & 3.1387 & 3.1265 \\
\hline 0.068499 & 3.2135 & 3.1930 & 3.1556 & 3.1690 & 3.1768 & 3.1874 & 3.1869 & 3.1866 & 3.1817 \\
\hline 0.078365 & 3.2646 & 3.2445 & 3.2029 & 3.2150 & 3.2291 & 3.2380 & 3.2267 & 3.2389 & 3.2175 \\
\hline 0.088250 & 3.2958 & 3.2727 & 3.2437 & 3.2450 & 3.2611 & 3.2718 & 3.2620 & 3.2702 & 3.2585 \\
\hline 0.098156 & 3.3349 & 3.3043 & 3.2664 & 3.2783 & 3.2918 & 3.3040 & 3.2952 & 3.2916 & 3.2823 \\
\hline m & \multicolumn{9}{|c|}{$30 \%$ EG } \\
\hline 0.009608 & 3.0161 & 2.9974 & 2.9648 & 2.9528 & 2.9460 & 2.9483 & 2.9469 & 2.9352 & 2.9340 \\
\hline 0.019236 & 3.1330 & 3.1234 & 3.0996 & 3.0877 & 3.0845 & 3.0802 & 3.0711 & 3.0722 & 3.0928 \\
\hline 0.028882 & 3.2346 & 3.2183 & 3.1975 & 3.1934 & 3.1821 & 3.1898 & 3.1890 & 3.1841 & 3.1848 \\
\hline 0.038548 & 3.3423 & 3.3380 & 3.3084 & 3.3035 & 3.3002 & 3.3085 & 3.3105 & 3.3058 & 3.3042 \\
\hline 0.048234 & 3.4417 & 3.4215 & 3.4022 & 3.4040 & 3.4004 & 3.3937 & 3.3917 & 3.3894 & 3.3887 \\
\hline 0.057939 & 3.5079 & 3.4878 & 3.4667 & 3.4605 & 3.4578 & 3.4716 & 3.4598 & 3.4554 & 3.4687 \\
\hline 0.067663 & 3.5595 & 3.5457 & 3.5200 & 3.5250 & 3.5156 & 3.5289 & 3.5232 & 3.5089 & 3.5245 \\
\hline 0.077407 & 3.5991 & 3.5927 & 3.5603 & 3.5689 & 3.5549 & 3.5679 & 3.5609 & 3.5516 & 3.5697 \\
\hline 0.087171 & 3.6366 & 3.6248 & 3.6056 & 3.6020 & 3.5941 & 3.6028 & 3.6010 & 3.5860 & 3.5936 \\
\hline 0.096954 & 3.6621 & 3.6569 & 3.6324 & 3.6323 & 3.6253 & 3.6365 & 3.6291 & 3.6228 & 3.6303 \\
\hline
\end{tabular}

Table 4. Thermodynamic functions of first dissociation constants $\mathbf{p} \mathbf{K}_{\mathbf{1}}$ at different temperatures obtained by (L.S.M.) with other constants in 10, 20, and 30\% Ethylene glycol-water mixtures.

\begin{tabular}{|c|c|c|c|c|c|c|c|}
\hline \multicolumn{8}{|c|}{$10 \%$ EG } \\
\hline $\mathbf{T} / \mathbf{K}$ & \multicolumn{3}{|c|}{$\beta \pm \sigma_{\beta}$} & \multicolumn{3}{|c|}{$\mathrm{pK}_{1} \pm \sigma_{\mathrm{pK}_{1}}$} & \multirow{2}{*}{$\begin{array}{c}\mathbf{R}^{\mathbf{2}} \\
0.9514\end{array}$} \\
\hline 278.15 & 3.5281 & \pm & 0.28221 & 2.6790 & \pm & 0.03587 & \\
\hline 283.15 & 3.5395 & \pm & 0.94756 & 2.6525 & \pm & 0.03746 & 0.9476 \\
\hline 288.15 & 3.5510 & \pm & 0.95654 & 2.6117 & \pm & 0.03409 & 0.9565 \\
\hline 293.15 & 3.6341 & \pm & 0.95018 & 2.6082 & \pm & 0.03752 & 0.9502 \\
\hline 298.15 & 3.7203 & \pm & 0.94364 & 2.6073 & \pm & 0.04101 & 0.9436 \\
\hline 303.15 & 3.7743 & \pm & 0.30965 & 2.6056 & \pm & 0.03961 & 0.9489 \\
\hline 308.15 & 3.7422 & \pm & 0.3426 & 2.6043 & \pm & 0.04396 & 0.9372 \\
\hline 313.15 & 3.7659 & \pm & 0.36762 & 2.6031 & \pm & 0.04732 & 0.9292 \\
\hline 318.15 & 3.687 & \pm & 0.36433 & 2.6026 & \pm & 0.04708 & 278.15 \\
\hline \multicolumn{8}{|c|}{$20 \%$ EG } \\
\hline 278.15 & 3.7340 & \pm & 0.23429 & 2.8983 & \pm & 0.02887 & 0.9696 \\
\hline 283.15 & 3.4778 & \pm & 0.95345 & 2.8746 & \pm & 0.03354 & 0.9535 \\
\hline 288.15 & 3.4606 & \pm & 0.95253 & 2.8317 & \pm & 0.03378 & 0.9525 \\
\hline 293.15 & 3.5276 & \pm & 0.94731 & 2.8172 & \pm & 0.03643 & 0.9473 \\
\hline 298.15 & 3.5029 & \pm & 0.94541 & 2.796 & \pm & 0.03696 & 0.9454 \\
\hline 303.15 & 5.5736 & \pm & 0.30580 & 2.7932 & \pm & 0.03807 & 0.9447 \\
\hline 308.15 & 3.5428 & \pm & 0.29927 & 2.7903 & \pm & 0.03733 & 0.946 \\
\hline 313.15 & 3.59 & \pm & 0.30032 & 2.7894 & \pm & 0.03753 & 0.947 \\
\hline 318.15 & 3.6285 & \pm & 0.33825 & 2.7888 & \pm & 0.04238 & 0.935 \\
\hline \multicolumn{8}{|c|}{$30 \%$ EG } \\
\hline 278.15 & 3.7072 & \pm & 0.307188 & 3.0150 & \pm & 0.03719 & 0.9480 \\
\hline 283.15 & 3.7458 & \pm & 0.952117 & 2.9979 & \pm & 0.03598 & 0.9521 \\
\hline 288.15 & 3.7626 & \pm & 0.949920 & 2.9708 & \pm & 0.03703 & 0.9499 \\
\hline 293.15 & 3.8385 & \pm & 0.947081 & 2.9596 & \pm & 0.03891 & 0.9471 \\
\hline 298.15 & 3.8146 & \pm & 0.944996 & 2.9549 & \pm & 0.03950 & 0.9450 \\
\hline 303.15 & 3.8819 & \pm & 0.327071 & 2.9539 & \pm & 0.03976 & 0.9463 \\
\hline 308.15 & 3.8584 & \pm & 0.327473 & 2.9516 & \pm & 0.03984 & 0.9455 \\
\hline 313.15 & 3.8189 & \pm & 0.334330 & 2.9484 & \pm & 0.04071 & 0.9422 \\
\hline 318.15 & 3.8529 & \pm & 0.337036 & 2.9519 & \pm & 0.04112 & 0.9423 \\
\hline
\end{tabular}

\section{Calculation of ionic strength and second dissociation constant}

Ionic strength $\mathrm{I}_{2}$ of the solution of the cell B is in the table (5) that is calculated from the equation (5).

$I_{2}=m_{1}+3 m_{2}+m_{3}$

From dissociation of $\mathrm{HMal}^{-}$

$\frac{E_{B^{-}-E_{m}^{0}}^{0}}{K}-\log \frac{m_{\mathrm{HMal}^{-}} \mathbf{m}_{\mathrm{Cl}^{-}}}{m_{\mathrm{Mal}^{-2}}}+\frac{2 \mathrm{~A} \sqrt{I_{2}}}{1+\mathrm{Ba}^{0} \sqrt{\mathrm{I}_{2}}}=-\beta \mathrm{I}_{2}-\log \mathrm{K}_{1}$

Where $E_{B}$ is the emf of the cell $B, E_{m}^{0}$ is the standard emf of $\mathrm{Ag}-\mathrm{AgCl}$ electrode, $(\mathrm{m})$ is the molality. The apparent second dissociation constant $\overline{\mathrm{K}}_{2},\left(-\log \mathrm{K}_{2}\right)$ of malonic acid from electromotive force of the cell $\mathrm{B}$ in different molal concentration and temperatures is obtained from the left hand side of eq (6)

The plot of $\mathrm{pK}_{2}$ is linear with $\mathrm{I}_{2}$ when $\mathrm{a}^{0}$ is taken to be 4.3 at each temperature. The thermodynamic values $\mathrm{pK}_{2}$ are listed in tables (6), the value of $\mathrm{pK}_{2}$ reflect a larger increase (larger decrease in acidity of malonic acid ) as mole fraction of Ethyle glycol increases. This may be due to the mono anion bering surrounded by the larger and highly viscous Ethylene glycol molecules. This slows down the second ionization step (decreases acidity of the acid) as mole fraction of ethylene glycol increases. The thermodynamic functions of the second dissociation constants $\mathrm{pK}_{2}$ at different temperatures obtained by (L.S.M.) with other constants in X\% Ethylene glycol-water 
mixtures show at the table (7). The $\mathrm{pK}_{1}$ and $\mathrm{pK}_{2}$ values decrease with the increase of temperature, the $\mathrm{pK}_{1}$ is less temperature dependent than $\mathrm{pK}_{2}$. This is referred to the higher heat of the acid dissociation in the second stage of the dissociation as compared with the first stage as shown in table (8).

Table (5) Ionic strength $I_{2}$ at different molal concentrations for the cell B in $10 \%$ Ethylene glycol-water mixtures.

\begin{tabular}{|c|c|c|c|c|c|c|c|c|}
\hline \multicolumn{3}{|c|}{$\mathbf{1 0 \%}$ EG } & \multicolumn{3}{|c|}{$\mathbf{2 0 \%}$ EG } & \multicolumn{3}{c|}{$\mathbf{3 0 \%}$ EG } \\
\hline $\mathbf{m}$ & $\mathbf{I}_{\mathbf{2}}$ & $\mathbf{I}_{\mathbf{2}}^{\mathbf{1 / 2}}$ & $\mathbf{~ m}$ & $\mathbf{I}_{\mathbf{2}}$ & $\mathbf{I}_{\mathbf{2}}^{\mathbf{1 / 2}}$ & $\mathbf{~ m}$ & $\mathbf{I}_{\mathbf{2}}$ & $\mathbf{I}_{\mathbf{2}}^{\mathbf{1 / 2}}$ \\
\hline 0.009979 & 0.0499 & 0.22339 & 0.009726 & 0.04864 & 0.220543 & 0.009608 & 0.0480488 & 0.2192 \\
\hline 0.019979 & 0.09993 & 0.31612 & 0.019472 & 0.09739 & 0.312079 & 0.019236 & 0.0962099 & 0.31018 \\
\hline 0.03 & 0.15008 & 0.3874 & 0.029238 & 0.14626 & 0.382443 & 0.028882 & 0.1444836 & 0.38011 \\
\hline 0.040042 & 0.20035 & 0.4476 & 0.039023 & 0.19525 & 0.441869 & 0.038548 & 0.1928703 & 0.43917 \\
\hline 0.050104 & 0.25074 & 0.50074 & 0.048829 & 0.24435 & 0.494317 & 0.048234 & 0.2413705 & 0.49129 \\
\hline 0.060188 & 0.30125 & 0.54886 & 0.058654 & 0.29357 & 0.541819 & 0.057939 & 0.2899846 & 0.5385 \\
\hline 0.070292 & 0.35189 & 0.5932 & 0.068499 & 0.3429 & 0.58558 & 0.067663 & 0.3387131 & 0.58199 \\
\hline 0.080418 & 0.40265 & 0.63455 & 0.078365 & 0.39236 & 0.626384 & 0.077407 & 0.3875564 & 0.62254 \\
\hline 0.090565 & 0.45354 & 0.67345 & 0.08825 & 0.44193 & 0.664777 & 0.087171 & 0.4365149 & 0.66069 \\
\hline 0.100733 & 0.50455 & 0.71032 & 0.098156 & 0.49162 & 0.701155 & 0.096954 & 0.4855892 & 0.69684 \\
\hline
\end{tabular}

Table 6. Appurant second dissociation constant $\mathbf{p} \overline{\mathbf{K}}_{\mathbf{2}}$ at different temperatures and molal concentrations in 10,20 , and $30 \%$ Ethylene glycol-water mixtures.

\begin{tabular}{|c|c|c|c|c|c|c|c|c|c|}
\hline \multicolumn{10}{|c|}{$10 \%$ EG } \\
\hline Concentration & \multicolumn{9}{|c|}{ Temperature ${ }^{\circ} \mathrm{C}$} \\
\hline $\mathbf{m}$ & 278.15 & 283.15 & 288.15 & 293.15 & 298.15 & 303.15 & 308.15 & 313.15 & 318.15 \\
\hline 0.009979 & 8.1899 & 8.1444 & 8.0861 & 8.0646 & 8.0470 & 8.0327 & 8.0179 & 8.0026 & 7.9746 \\
\hline 0.019979 & 7.7348 & 7.6821 & 7.6277 & 7.6148 & 7.6022 & 7.5945 & 7.5811 & 7.5730 & 7.5478 \\
\hline 0.030000 & 7.3835 & 7.3398 & 7.2925 & 7.2725 & 7.2648 & 7.2604 & 7.2467 & 7.2520 & 7.2264 \\
\hline 0.040042 & 7.1796 & 7.1430 & 7.0957 & 7.0911 & 7.0796 & 7.0700 & 7.0642 & 7.0632 & 7.0405 \\
\hline 0.050104 & 7.0812 & 7.0466 & 6.9910 & 6.9782 & 6.9757 & 6.9748 & 6.9628 & 6.9609 & 6.9464 \\
\hline 0.060188 & 7.0032 & 6.9582 & 6.9153 & 6.9044 & 6.8952 & 6.8897 & 6.8763 & 6.8831 & 6.8625 \\
\hline 0.070292 & 6.9301 & 6.8800 & 6.8429 & 6.8339 & 6.8301 & 6.8182 & 6.8050 & 6.8123 & 6.7887 \\
\hline 0.080418 & 6.8555 & 6.8201 & 6.7745 & 6.7642 & 6.7607 & 6.7491 & 6.7380 & 6.7441 & 6.7273 \\
\hline 0.090565 & 6.7993 & 6.7589 & 6.7101 & 6.7036 & 6.7038 & 6.6925 & 6.6849 & 6.6817 & 6.6651 \\
\hline 0.100733 & 6.7386 & 6.7039 & 6.6572 & 6.6475 & 6.6464 & 6.6321 & 6.6248 & 6.6301 & 6.6154 \\
\hline $\mathbf{m}$ & \multicolumn{9}{|c|}{$20 \%$ EG } \\
\hline 0.009726 & 8.6250 & 8.5399 & 8.4860 & 8.4449 & 8.4031 & 8.3926 & 8.3632 & 8.3485 & 8.3361 \\
\hline 0.019472 & 8.1330 & 8.0539 & 7.9952 & 7.9618 & 7.9233 & 7.9200 & 7.8966 & 7.8773 & 7.8715 \\
\hline 0.029238 & 7.8423 & 7.7675 & 7.7095 & 7.6734 & 7.6397 & 7.6353 & 7.6229 & 7.6089 & 7.5908 \\
\hline 0.039023 & 7.6444 & 7.5712 & 7.5095 & 7.4872 & 7.4503 & 7.4457 & 7.4334 & 7.4240 & 7.4136 \\
\hline 0.048829 & 7.5445 & 7.4752 & 7.4103 & 7.3884 & 7.3572 & 7.3462 & 7.3345 & 7.3287 & 7.3184 \\
\hline 0.058654 & 7.4632 & 7.3853 & 7.3243 & 7.3027 & 7.2689 & 7.2716 & 7.2569 & 7.2433 & 7.2334 \\
\hline 0.068499 & 7.3867 & 7.3110 & 7.2504 & 7.2240 & 7.1992 & 7.1955 & 7.1797 & 7.1712 & 7.1616 \\
\hline 0.078365 & 7.3250 & 7.2441 & 7.1909 & 7.1613 & 7.1352 & 7.1252 & 7.1165 & 7.1082 & 7.0941 \\
\hline 0.088250 & 7.2654 & 7.1884 & 7.1231 & 7.0974 & 7.0736 & 7.0622 & 7.0572 & 7.0460 & 7.0370 \\
\hline 0.098156 & 7.2049 & 7.1283 & 7.0668 & 7.0415 & 7.0215 & 7.0103 & 7.0041 & 6.9931 & 6.9843 \\
\hline $\mathbf{m}$ & \multicolumn{9}{|c|}{$30 \%$ EG } \\
\hline 0.009608 & 9.0974 & 9.0477 & 8.9821 & 8.9546 & 8.9209 & 8.8937 & 8.8674 & 8.8249 & 8.8202 \\
\hline 0.019236 & 8.5924 & 8.5650 & 8.5035 & 8.4700 & 8.4419 & 8.4242 & 8.3990 & 8.3668 & 8.3515 \\
\hline 0.028882 & 8.3243 & 8.2885 & 8.2328 & 8.2000 & 8.1739 & 8.1620 & 8.1392 & 8.1060 & 8.0989 \\
\hline 0.038548 & 8.1323 & 8.0982 & 8.0424 & 8.0146 & 7.9832 & 7.9730 & 7.9454 & 7.9235 & 7.9162 \\
\hline 0.048234 & 8.0311 & 7.9885 & 7.9454 & 7.9216 & 7.8819 & 7.8723 & 7.8584 & 7.8255 & 7.8186 \\
\hline 0.057939 & 7.9411 & 7.9025 & 7.8581 & 7.8296 & 7.8073 & 7.7947 & 7.7664 & 7.7454 & 7.7309 \\
\hline 0.067663 & 7.8704 & 7.8267 & 7.7810 & 7.7581 & 7.7343 & 7.7222 & 7.6977 & 7.6754 & 7.6659 \\
\hline 0.077407 & 7.8035 & 7.7620 & 7.7166 & 7.6957 & 7.6688 & 7.6538 & 7.6346 & 7.6127 & 7.6019 \\
\hline 0.087171 & 7.7477 & 7.7101 & 7.6613 & 7.6304 & 7.6073 & 7.5928 & 7.5756 & 7.5492 & 7.5404 \\
\hline 0.096954 & 7.6892 & 7.6572 & 7.6035 & 7.5782 & 7.5536 & 7.5411 & 7.5259 & 7.5030 & 7.4896 \\
\hline
\end{tabular}

Table 7. Thermodynamic functions of the second dissociation constants $\mathbf{p} \mathbf{K}_{2}$ at different temperatures obtained by (L.S.M.) with other constants in $10 \%$ Ethylene glycol-water mixtures.

\begin{tabular}{|c|c|c|c|c|c|c|c|}
\hline \multicolumn{8}{|c|}{$10 \%$ EG } \\
\hline \multirow{2}{*}{$\frac{\mathbf{T} / \mathbf{K}}{278.15}$} & \multicolumn{3}{|c|}{$\beta \pm \sigma_{\beta}$} & \multicolumn{3}{|c|}{$\mathrm{pK}_{2} \pm \sigma_{\mathrm{pK}_{2}}$} & \multirow{2}{*}{$\begin{array}{l}\mathbf{R}^{\mathbf{2}} \\
0.8399\end{array}$} \\
\hline & -2.7652 & \pm & 0.42689 & 7.9541 & \pm & 0.133296 & \\
\hline 283.15 & -2.7443 & \pm & 0.83932 & 7.9065 & \pm & 0.132554 & 0.8393 \\
\hline 288.15 & -2.7211 & \pm & 0.84071 & 7.8517 & \pm & 0.130759 & 0.8517 \\
\hline 293.15 & -2.6984 & \pm & 0.84124 & 7.8336 & \pm & 0.129415 & 0.8412 \\
\hline 298.15 & -2.6653 & \pm & 0.83986 & 7.8175 & \pm & 0.128486 & 0.8399 \\
\hline 303.15 & -2.6742 & \pm & 0.40388 & 7.8108 & \pm & 0.12611 & 0.8457 \\
\hline
\end{tabular}




\begin{tabular}{|c|c|c|c|c|c|c|c|}
\hline 308.15 & -2.6625 & \pm & 0.40298 & 7.7963 & \pm & 0.12583 & 0.8451 \\
\hline 313.15 & -2.6317 & \pm & 0.39337 & 7.7879 & \pm & 0.122827 & 0.8484 \\
\hline 318.15 & -2.6059 & \pm & 0.39172 & 7.76 & \pm & 0.122313 & 0.8469 \\
\hline \multicolumn{8}{|c|}{$20 \%$ EG } \\
\hline 278.15 & -2.7420 & \pm & 0.42206 & 8.3822 & \pm & 0.128417 & 0.8407 \\
\hline 283.15 & -2.7366 & \pm & 0.84521 & 8.3038 & \pm & 0.125983 & 0.8452 \\
\hline 288.15 & -2.7468 & \pm & 0.84368 & 8.2467 & \pm & 0.127188 & 0.8437 \\
\hline 293.15 & -2.7203 & \pm & 0.84697 & 8.2112 & \pm & 0.124389 & 0.8470 \\
\hline 298.15 & -2.6747 & \pm & 0.84249 & 8.1678 & \pm & 0.124407 & 0.8425 \\
\hline 303.15 & -2.6838 & \pm & 0.40311 & 8.1636 & \pm & 0.122652 & 0.8471 \\
\hline 308.15 & -2.6417 & \pm & 0.39644 & 8.1382 & \pm & 0.120624 & 0.8473 \\
\hline 313.15 & -2.6278 & \pm & 0.39341 & 8.1229 & \pm & 0.119699 & 0.8480 \\
\hline 318.15 & -2.6236 & \pm & 0.39432 & 8.111 & \pm & 0.119977 & 0.8470 \\
\hline \multicolumn{8}{|c|}{$30 \%$ EG } \\
\hline 278.15 & -2.7476 & \pm & 0.41965 & 8.8542 & \pm & 0.126123 & 0.8427 \\
\hline 283.15 & -2.7433 & \pm & 0.84534 & 8.8147 & \pm & 0.124685 & 0.8453 \\
\hline 288.15 & -2.7091 & \pm & 0.84843 & 8.7537 & \pm & 0.121673 & 0.8484 \\
\hline 293.15 & -2.6957 & \pm & 0.84696 & 8.7227 & \pm & 0.121761 & 0.8470 \\
\hline 298.15 & -2.6765 & \pm & 0.84476 & 8.6896 & \pm & 0.121916 & 0.8448 \\
\hline 303.15 & -2.6603 & \pm & 0.39526 & 8.6710 & \pm & 0.118793 & 0.8499 \\
\hline 308.15 & -2.6393 & \pm & 0.39654 & 8.6434 & \pm & 0.119178 & 0.8470 \\
\hline 313.15 & -2.6040 & \pm & 0.38858 & 8.6062 & \pm & 0.116786 & 0.8488 \\
\hline 318.15 & -2.6121 & \pm & 0.38942 & 8.5986 & \pm & 0.117037 & 0.8490 \\
\hline
\end{tabular}

Table 8. $\Delta \mathbf{p K}$ and the ratio $\mathbf{K}_{\mathbf{1}} / \mathbf{K}_{\mathbf{2}}$ of malonic acid at different temperatures in 10,20, and 30\% Ethylene glycol-water mixtures.

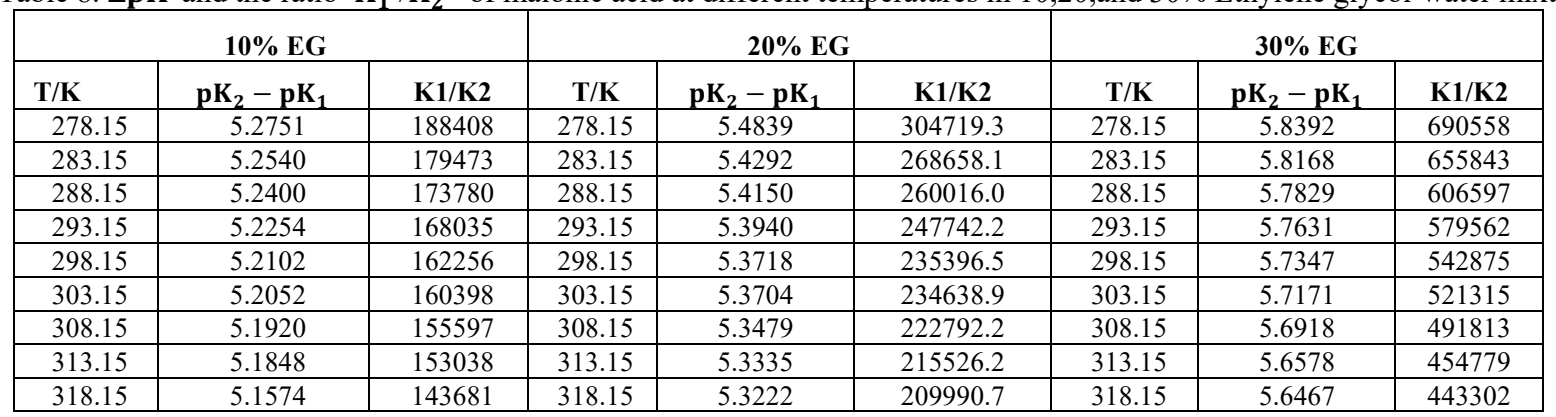

\section{Thermodynamic Quantities of Dissociation}

$\mathrm{pK}=\frac{\mathrm{A}_{1}}{\mathrm{~T}}-\mathrm{A}_{2}+\mathrm{A}_{3} \mathrm{~T}$

The values of the first and second thermodynamic dissociation constant, $\left(p K_{i}\right)$ thus obtained at different temperatures were fitted to an equation of the type (7) by writing it in the form $\mathrm{T}$. $\mathrm{pK}=\mathrm{A}_{1}-\mathrm{A}_{2} \mathrm{~T}+\mathrm{A}_{3} \mathrm{~T}^{2}$

By giving the data of T.pK and $\mathrm{T}$ for the computer and by the (L.S.M.) method as shown in figure 1,2 . The value of constant $\left(A_{1}, A_{2}\right.$ and $\left.A_{3}\right)$ that is used to calculate thermodynamic quantities for the first and second dissociation obtained as follows[9].

a) The standard change in free energy, $\Delta \mathrm{G}_{\mathrm{i}}^{0}=2.3026 \mathrm{R}\left(\mathrm{A}_{1}-\right.$ $\mathrm{A}_{2} \mathrm{~T}+\mathrm{A}_{3} \mathrm{~T}^{2}$ )

b) The standard change in entropy, $\Delta \mathrm{S}_{\mathrm{i}}^{0}=2.3026 \mathrm{R}\left(\mathrm{A}_{2}\right.$ $2 \mathrm{~A}_{3} \mathrm{~T}$ )

c) The standard change in Heat content, $\Delta \mathrm{H}_{\mathrm{i}}^{0}=$ 2.3026R $\left(A_{1}-A_{3} T^{2}\right)$

(11) d) The standard change in Heat capacity, $\Delta \mathrm{Cp}_{\mathrm{i}}^{0}=$ $2.3026 \mathrm{R}\left(-2 \mathrm{~A}_{3} \mathrm{~T}\right)$

The value of thermodynamic functions for the first and second dissociation are summarized in table (9) and (10) respectively. The negative value of $\Delta \mathrm{S}_{i}^{0}$ for both dissociation processes increase as temperature increase, also it becomes more negative in mixture with higher $\mathrm{X} \%$ Ethylene glycol-water content, The value of $\Delta \mathrm{H}^{0}$ decreases as temperature increase and decreases with the increase of $\mathrm{X} \%$ Ethylene glycol-water. This can be the fact that the reaction becomes more endothermic in mixture with high $\mathrm{X} \%$ constants, The negative value of thermodynamic function standard heat capacity $\Delta \mathrm{Cp}^{0}$, increase with the increase of temperature and decreases with the increase of $\mathrm{X} \%$ Ethylene glycol-water mixtures, this is due to points of electrostatic interaction with the charged which leads to the orientation of the solvent molecules.

Table 9. Thermodynamic functions for dissociation at different temperatures from first dissociation constant for malonic acid in $10 \%$ Ethylene glycol-water mixtures

\begin{tabular}{|c|c|c|c|c|}
\hline \multicolumn{5}{|c|}{$10 \%$ EG } \\
\hline $\mathbf{T} / \mathbf{K}$ & $\begin{array}{c}\Delta \mathbf{G}_{\mathbf{1}} \\
\left(\mathrm{J} . \mathbf{m o l}^{-\mathbf{1}}\right)\end{array}$ & $\begin{array}{c}\Delta \mathbf{S}_{\mathbf{1}}^{\mathbf{1}} \\
\left(\mathrm{J} . \mathbf{~ m o l}^{-\mathbf{1}}\right)\end{array}$ & $\begin{array}{c}\Delta \mathbf{H}_{\mathbf{1}}^{\mathbf{1}} \\
\left(\mathrm{J} . \mathbf{m o l}^{-\mathbf{1}}\right)\end{array}$ & $\begin{array}{c}\Delta \mathbf{C p}_{\mathbf{1}}^{\mathbf{1}} \\
\left(\mathrm{J} . \mathrm{mol}^{-\mathbf{1}}\right)\end{array}$ \\
\hline 278.15 & 14224.24 & -21.5025 & 8243.314 & -275.837 \\
\hline 283.15 & 14344.15 & -26.4610 & 6851.731 & -280.796 \\
\hline 288.15 & 14488.85 & -31.4194 & 5435.356 & -285.754 \\
\hline 293.15 & 14658.35 & -36.3778 & 3994.190 & -290.713 \\
\hline 298.15 & 14852.63 & -41.3362 & 2528.230 & -295.671 \\
\hline 303.15 & 15071.71 & -46.2947 & 1037.479 & -300.629 \\
\hline
\end{tabular}




\begin{tabular}{|l|r|r|r|r|}
\hline 308.15 & 15315.58 & -51.2531 & -478.064 & -305.588 \\
\hline 313.15 & 15584.24 & -56.2115 & -2018.40 & -310.546 \\
\hline 318.15 & 15877.69 & -61.1700 & -3583.53 & -315.505 \\
\hline \multicolumn{5}{|c|}{$\mathbf{2 0 \% \text { EG }}$} \\
\hline 278.15 & 15401.39 & -15.0413 & 11217.66 & -329.088 \\
\hline 283.15 & 15491.38 & -20.9569 & 9557.432 & -335.003 \\
\hline 288.15 & 15610.96 & -26.8726 & 7867.626 & -340.919 \\
\hline 293.15 & 15760.11 & -32.7882 & 6148.241 & -346.835 \\
\hline 298.15 & 15938.84 & -38.7039 & 4399.278 & -352.75 \\
\hline 303.15 & 16147.15 & -44.6195 & 2620.737 & -358.666 \\
\hline 308.15 & 16385.03 & -50.5352 & 812.6182 & -364.582 \\
\hline 313.15 & 16652.5 & -56.4508 & -1025.08 & -370.497 \\
\hline 318.15 & 16949.54 & -62.3665 & -2892.36 & -376.413 \\
\hline \multicolumn{5}{|c|}{$\mathbf{3 0 \%}$ EG } \\
\hline 278.15 & 16119.63 & -33.5825 & 6778.65 & -215.132 \\
\hline 283.15 & 16297.21 & -37.4497 & 5693.323 & -218.999 \\
\hline 288.15 & 16494.13 & -41.3169 & 4588.66 & -222.866 \\
\hline 293.15 & 16710.38 & -45.1841 & 3464.661 & -226.733 \\
\hline 298.15 & 16945.97 & -49.0513 & 2321.326 & -230.601 \\
\hline 303.15 & 17200.89 & -52.9185 & 1158.656 & -234.468 \\
\hline 308.15 & 17475.15 & -56.7857 & -23.3512 & -238.335 \\
\hline 313.15 & 17768.75 & -60.6529 & -1224.69 & -242.202 \\
\hline 318.15 & 18081.68 & -64.5201 & -2445.37 & -246.069 \\
\hline
\end{tabular}

Table 10. Thermodynamic functions for dissociation at different temperatures from second dissociation constant for malonic acid in 10,20 , and $30 \%$ Ethylene glycol-water mixtures

\begin{tabular}{|c|c|c|c|c|}
\hline \multicolumn{5}{|c|}{$10 \% \mathrm{EG}$} \\
\hline $\mathbf{T} / \mathbf{K}$ & $\begin{array}{c}\Delta \mathbf{G}_{2}^{0} \\
\left(\text { J. } \mathbf{m o l}^{-1}\right)\end{array}$ & $\begin{array}{c}\Delta S_{2}^{0} \\
\left(\text { J. } \mathrm{mol}^{-1}\right)\end{array}$ & $\begin{array}{c}\Delta \mathbf{H}_{2}^{0} \\
\left(\text { J. } \mathbf{m o l}^{-1}\right)\end{array}$ & $\begin{array}{c}\Delta \mathrm{Cp}_{2}^{0} \\
\left.\text { (J. mol }{ }^{-1}\right)\end{array}$ \\
\hline 278.15 & 42331.56 & -106.805 & 12623.77 & -272.642 \\
\hline 283.15 & 42877.84 & -111.706 & 11248.31 & -277.543 \\
\hline 288.15 & 43448.62 & -116.607 & 9848.34 & -282.444 \\
\hline 293.15 & 44043.90 & -121.508 & 8423.866 & -287.345 \\
\hline 298.15 & 44663.70 & -126.409 & 6974.887 & -292.246 \\
\hline 303.15 & 45307.99 & -131.31 & 5501.404 & -297.147 \\
\hline 308.15 & 45976.79 & -136.211 & 4003.415 & -302.048 \\
\hline 313.15 & 46670.10 & -141.112 & 2480.921 & -306.949 \\
\hline 318.15 & 47387.91 & -146.013 & 933.9224 & -311.85 \\
\hline \multicolumn{5}{|c|}{$20 \%$ EG } \\
\hline 278.15 & 44511.74 & -86.8008 & 20368.09 & -474.994 \\
\hline 283.15 & 44967.09 & -95.3393 & 17971.77 & -483.532 \\
\hline 288.15 & 45465.13 & -103.878 & 15532.76 & -492.071 \\
\hline 293.15 & 46005.86 & -112.416 & 13051.06 & -500.609 \\
\hline 298.15 & 46589.29 & -120.955 & 10526.67 & -509.148 \\
\hline 303.15 & 47215.41 & -129.493 & 7959.586 & -517.686 \\
\hline 308.15 & 47884.22 & -138.032 & 5349.809 & -526.225 \\
\hline 313.15 & 48595.72 & -146.57 & 2697.34 & -534.763 \\
\hline 318.15 & 49349.92 & -155.108 & 2.177812 & -543.302 \\
\hline \multicolumn{5}{|c|}{$30 \%$ EG } \\
\hline 278.15 & 47203 & -116.355 & 14838.76 & -200.222 \\
\hline 283.15 & 47793.77 & -119.955 & 13828.66 & -203.821 \\
\hline 288.15 & 48402.55 & -123.554 & 12800.55 & -207.42 \\
\hline 293.15 & 49029.31 & -127.153 & 11754.46 & -211.019 \\
\hline 298.15 & 49674.07 & -130.752 & 10690.36 & -214.618 \\
\hline 303.15 & 50336.83 & -134.351 & 9608.273 & -218.218 \\
\hline
\end{tabular}




\begin{tabular}{|l|l|l|l|l|}
\hline 308.15 & 51017.59 & -137.95 & 8508.188 & -221.817 \\
\hline 313.15 & 51716.33 & -141.55 & 7390.106 & -225.416 \\
\hline 318.15 & 52433.08 & -145.149 & 6254.029 & -229.015 \\
\hline
\end{tabular}

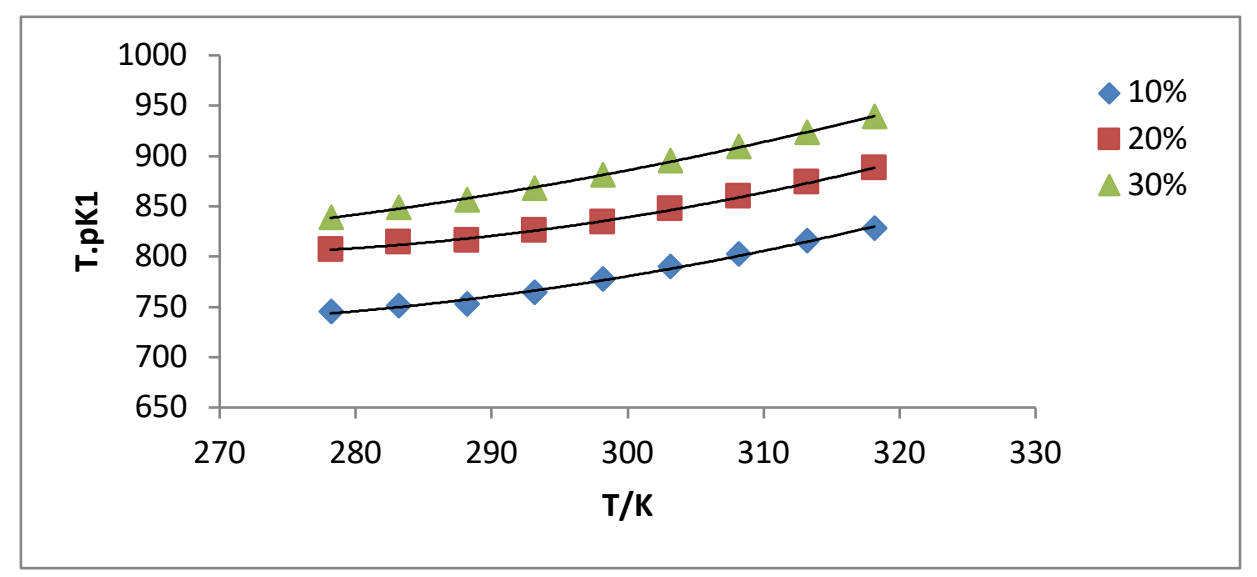

Fig. 1. change of T.pK1 with the temperature $\mathrm{T} / \mathrm{K}$ in a different composition of Ethylene glycol-water mixtures

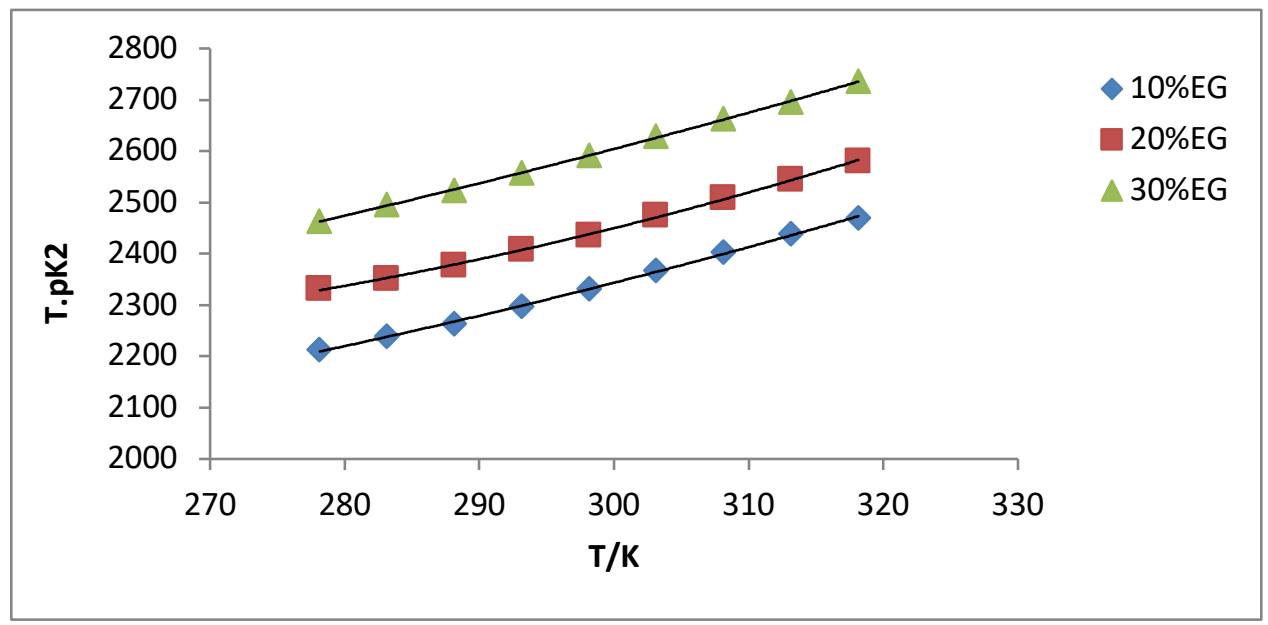

Fig. 2. change of T.pK2 with the temperature $\mathrm{T} / \mathrm{K}$ in a different composition of Ethylene glycol-water mixtures

\section{CONCLUSION}

The ratio $\mathrm{K}_{1} / \mathrm{K}_{2}$ decreases with the increase of the temperature because the water is attached by strong hydrogen bond with the carboxylate and weak bond with the carboxyl group. Weakness is caused in the intra-hydrogen bonding that effect for the ratio $\mathrm{K}_{1} / \mathrm{K}_{2}$ when the $\mathrm{X} \%$ Ethylene glycol is added to the water. The ethylene glycol molecule is attached by hydrogen bonding with the water and this causes decrease of water attached with the acid ion leading to increase intra-hydrogen bond force following the increase in the value of $\mathrm{K}_{1} / \mathrm{K}_{2}$.

The positive values of $\Delta G_{i}^{0}$ and $\Delta H_{i}^{0}$ indicate that nonspontaneous and endothermic cell reaction, and the negative values of $\Delta S_{i}^{0}$ revealing that the entropy is not in favor of cell reaction.

\section{REFERENCES:}

[1] E. O. Al-Tamimi and N. A. Ali, "Synthesis of New Poly ( Subs- Vinyl Malonate Amide ) from Malonic Acid," Int. J. Sci. Res., vol. 6, no. 8, pp. 1883-1888, 2017.

[2] F. A. Carey, Organic Chemistry. McGraw-Hill, Inc. America, 1880.

[3] T. D. Orlova, P. A. Romodanovskii, N. G. Dmitrieva, and S. N. Gridchin, "The Heat Effects of Malonic Acid
Dissociation," Russ. J. Phys. Chem. A., vol. 82, no. 4, pp. 681-683, 2008.

[4] J. Moreno and R. Peinado, "Carboxylic Acids, structure and properties, chapter 8," in Enological Chemistry," Elsevier, no. 5, pp. 109-120, 2012.

[5] T. Awad, G. Genidy, and G. A. Yahya, "Development of Novel Potentiometric Sensors for Determination of Lidocaine Hydrochloride in Pharmaceutical Preparations, Serum and Urine Samples," Iran. J. Pharm. Res., vol. 16, pp. 498-512, 2017.

[6] P. Thakur, J. N. Mathur, R. C. Moore, and G. R. Choppin, "Thermodynamics and dissociation constants of carboxylic acids at high ionic strength and temperature," Inorganica Chim. Acta, vol. 360, no. 12, pp. 3671-3680, 2007.

[7] K. Sue, F. Ouchi, K. Minami, and K. Arai, "Determination of Carboxylic Acid Dissociation Constants to $350{ }^{\circ} \mathrm{C}$ at 23 MPa by Potentiometric pH Measurements," J. Chem. Eng. Data, vol. 49, no. 5, pp. 1359-1363, 2004.

[8] Z. Qiang and C. Adams, "Potentiometric determination of acid dissociation constants $(\mathrm{pKa})$ for human and veterinary antibiotics," Water Res., vol. 38, pp. 2874-2890, 2004.

[9] A. K. Kralj, "Calculating the Dissociation Constant of Weak Electrolytes by Measuring the Conductivity," Int. J. Nonlinear Sci. Numer. Simulation., vol. 10, no. 7, pp. 965975, 2009.

[10] M. L. Kilpatrick, "Errors in the determination of the dissociation constant of a weak acid by the extrapolation method errors in the determination of the dissociation constant of a weak acid by the extrapolation method," $J$. 
Chem. Phys., vol. 8, pp. 306-313, 1940.

[11] D. D. Perrin, "Dissociation contants of inorganic acids and bases in aqueous solution," Pure Appl. Chem., vol. 20, no. 2, pp. 133-219, 1969.

[12] S. M. Moazzem Hossen, M. M. Hossain, M. Enamul, and G. Akteruzzaman, "Spectrophotometric Method for Determination of Dissociation Constant of weak acid like 2, 4-Dinitrophenol in 1-Propanol-Water Mixtures at $24.5 \pm 0.5^{\circ} \mathrm{C}$ S.," Der Pharma Chem., vol. 4, no. 4, pp. 1375 1384, 2012.

[13] L. E. Vidal Salgado and C. Vargas-Hernández, "Spectrophotometric Determination of the $\mathrm{pKa}$, Isosbestic Point and Equation of Absorbance vs . pH for a Universal pH Indicator," Am. J. Anal. Chem., vol. 5, pp. 1290-1301, 2014.

[14] K. P. Alter, J. L. Molloy, and E. D. Niemeyer, "Spectrophotometric Determination of the Dissociation Constant of an Acid - Base Indicator Using a Mathematical Deconvolution Technique," J. Chem. Educ., vol. 82, no. 11, pp. $1682-1685,2005$

[15] H. J. Ashby, H. B. Clarke, E. M. Crook, and S. P. Datta "Thermodynamic quantities for the dissociation equilibria of biologically important compounds," J. Biol. Chem., vol. 59, pp. 203-208, 1955.

[16] I. S. Majeed, "Dissociation constants of malonic acid in methyl urea + water mixture at different temperaturs"M.Sc. Thesis, University of Bagdad," 1979.

[17] I. S. Majeed and J. M. Saleh, "Acidic dissociation constants of malonic acid in N-methyl urea + water mixtures from e.m.f. measurements," Iraqi J. S., vol. 23, no. 3, 1982.

[18] M. K. Ridley, D. A. Plamer, D. J. and Wesolowski, and R. M. Kettler, "Cadimum Malonate complexation in aqueous sodium trifluoro methane sulfomate media to $75 \mathrm{C}$," J. Chem. S, vol. 27, no. 3, pp. 195-216, 2004

[19] I. P. and Jaakko and K. C. Arthur, "Reviews Re-evaluation of the First and Second Stoichiometric Dissociation Constants of Solutions with or without Potassium Chloride . 1. Estimation of the Parameters for the Hu 1 ckel Model Activity Coefficient Equations for Calculation of the Second," J. Chem. Eng. Data, vol. 51, pp. 777-784, 2006.

[20] Y. A. Fadeeva and L. P. Safonova, "Dissociation Constant of Acetic Acid in ( N, N-Dimethylformamide + Water ) Mixtures at the Temperature 298 . $15 \mathrm{~K}$, , J Solut. Chem, vol. 40, pp. 980-988, 2011.

[21] P. Y. Tishchenko, C. S. Wong, and W. K. Johnson,
"Measurements of Dissociation Constants of Carbonic Acid in Synthetic Seawater by Means of a Cell Without," J Solut. Chem, vol. 42, pp. 2168-2186, 2013.

[22] R. N. Roy et al., "Acid Dissociation Constants and Related Thermodynamic Functions of Protonated Nitrilotriethanol ( BIS-TRIS ) from ( 278.15 to 328 . 15 ) K," J. Biophys. Chem., vol. 5, pp. 118-124, 2014.

[23] R. N. Roy et al., "Thermodynamics of the Second Stage Dissociation Step ( p K 2 ) of Buffer Monosodium 1,4Piperazinediethanesulfonate from (278.15 to 328.15$) \mathrm{K}$, $J$. Biophys. Chem., vol. 5, pp. 91-98, 2014.

[24] E. N. Tsurko, "Thermodynamic Analysis of Dissociation Functions of Valine at $293.15-318.15 \mathrm{~K}$ in Ethanol-Water Mixtures," J Solut. Chem., pp. 1313-1330, 2014.

[25] R. N. Roy et al., "Dissociation Constant of Acid Monosodium ( ADA ) from ( 278.15 to 328.15 ) K," Journal of Physical Chemistry, no. 4, pp. 73-79, 2014.

[26] K. K. Kundu and M. N. Das, "Standard Potentials of Ag$\mathrm{AgCI}$ and $\mathrm{Ag}-\mathrm{AgBr}$ Electrodes in Ethylene Glycol and Propylene Glycol at 3OOC . and Related Thermodynamic Quantities," J. Chem. Eng. Data, vol. 9, no. 1, pp. 9-11, 1964.

"http://www.docbrown.info/page01/ExIndChem/electroch emistry02.htm.".

[28] K. M. Shareef, "Association constants and transference numbers of some uni-univalent electrolytes in mixed solvents using electro chemical methods," $\mathrm{Ph}$. D. Thesis, University of Saddam, 1994.

[29] S. K. Lower, Electrochemistry, 2nd. editi. 1994.

[30] L. F. Grantham and D. S. Martin, "Kinetics of chloride exchange in aqueous chloride- tetrachloroplatinate ( II ) system," Iowa, 1954.

[31] D. T. Sawyer, A. Sobkowiak, and A. Sobkowiak, Electrochemistry for Chemists, 2nd ed. 1995.

[32] A. K. Covington, C. M. A. Brett, M. F. Camoes, and R. Naumann, "Measurement of $\mathrm{pH}$. definition, standards, and procedures," Pure Appl. Chem, vol. 74, no. 11, pp. 2169 2200, 2002.

[33] S. S. Ahmed and L. A. Jamil, "Standard Potential of the Silver-Silver Chloride Electrode in X\% Ethylene Glycol Water Mixtures at Different Temperatures," Int. Conf. Adv. Sci. Eng., pp. 403-408, 2018. 